\title{
Search for DIBs in Emission: MWC 922 - The Red Square Nebula
}

\author{
N. Wehres ${ }^{1,2} \dagger$, B. Ochsendorf ${ }^{3}$, J. Bally ${ }^{2}$, T. Snow ${ }^{1,4}$, V. Bierbaum ${ }^{1,2}$, \\ N. L. J. $\operatorname{Cox}^{5}$, L. Kaper ${ }^{6}$ and A. G. G. M. Tielens ${ }^{3}$ \\ ${ }^{1}$ Center for Astrophysics and Space Astronomy, 389 UCB, University of Colorado, \\ Boulder, CO 80309-0389, USA \\ email: Nadine1. Wehres@emory .edu \\ ${ }^{2}$ Department of Chemistry and Biochemistry, 215 UCB, University of Colorado, \\ Boulder, CO 80309-0215, USA \\ ${ }^{3}$ Leiden Observatory, Leiden University, PO Box 9513, 2300 RA Leiden, The Netherlands \\ ${ }^{4}$ Department of Astrophysical and Planetary Sciences, 391 UCB, University of Colorado, \\ Boulder, CO 80309-0391, USA \\ ${ }^{5}$ Instituut voor Sterrenkunde, KU Leuven, Celestijnenlaan 200D, 3001 Leuven, Belgium \\ ${ }^{6}$ Astronomical Institute Anton Pannekoek, University of Amsterdam, \\ Science Park 904, 1098 XH Amsterdam, The Netherlands
}

\begin{abstract}
This work focusses on MWC 922, the central object in the Red Square Nebula. We obtained low and medium resolution spectra of both, the central object and the surrounding nebula, using the DIS and TSpec spectrograph. The spectra show the whole spectral range between $\sim 3500$ Åup to $\sim 25000 \AA$. The central object shows a plethora of emission lines, including many Fe II and forbidden Fe [II] lines. Here, we present the inventory of the emission lines of the central object, MWC 922. Future work will comprise the identification of the nebula emission lines by using newly obtained X-Shooter spectra. That way we want to gain further insight into the physical and chemical conditions in this environment. A comparison of the Red Square to the Red Rectangle Nebula is anticipated and will guide our search for DIBs in emission.
\end{abstract}

Keywords. Red Square Nebula, MWC 922, DIBs in emission

\section{Introduction}

The Red Square Nebula is surrounding the B[e] emission line object MWC 922. The evolutionary stage of MWC 922 is still unclear, and both pre and post-main sequence evolutionary status have been suggested (Tuthill \& Lloyd 2007). The central object is mainly known for its infrared properties (Allen \& Swings 1976). Allen \& Swings (1976) give also a brief description of the optical properties of MWC 922 in their paper on B[e] stars with infrared excess. Whereas Fe II lines are mentioned absent in that paper, Rudy et al. (1992) published the IR spectrum of MWC 922 and noted unusually strong Fe II and [Fe II] lines. In turn, Rudy et al. (1992) mentioned the absence of the Paschen series as well as any other absorption features. Here, we present our spectra with higher spectral resolution and larger spectral coverage. Most of the stellar emission lines could be identified and are shown in Fig. 2 and listed in table 1.

$\dagger$ Present address: Department of Chemistry, Emory University, 1515 Dickey Drive, Atlanta, GA 30322, USA 


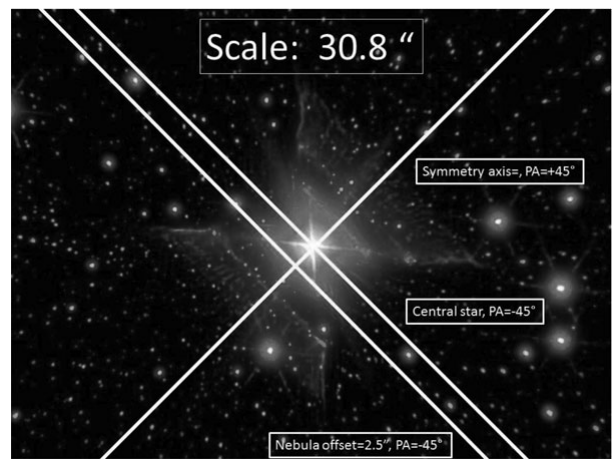

Figure 1. Slit positions used for obtaining the DIS and TSpec spectra. Image credit: Peter Tuthill and James Lloyd
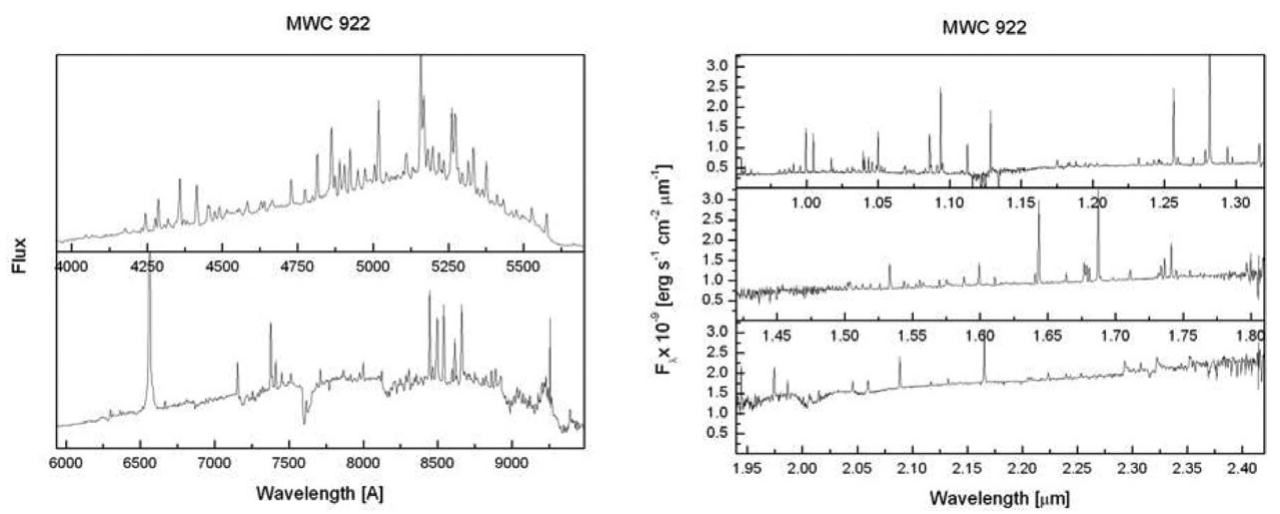

Figure 2. DIS (left panel) and TSpec (right panel) spectra of emission line object MWC 922.

\section{Observations}

The optical spectra were obtained using DIS (Dual Imaging Spectrograph), and the near-IR spectra were obtained using TSpec. Both spectrographs are mounted on the $3.5 \mathrm{~m}$ telescope at the APO. DIS was operated in his standard settings using a $1.2^{\prime \prime} \times 6^{\prime}$ slit and the B400/R300 gratings. This gives about $1.83 \AA \mathrm{pix}^{-1}$ and $2.31 \AA \mathrm{pix}^{-1}$ spectral resolution, and a coverage between 3500 and $7500 \AA$. The near-IR spectra were obtained using a slit dimension of $1.1^{\prime \prime} \times 43^{\prime \prime}$, giving a spectral resolution of $\frac{\lambda}{\Delta \lambda} \sim 3500$. The spectra cover the region between $9500 \AA$ and $25000 \AA$. The slit positions for both spectrographs are displayed in Fig. 1. We positioned the slit along the symmetry axis of the nebula as well as perpendicular to the symmetry axis. The PA of the Red Square Nebula is $46^{\circ}$. Not discussed here is the offset position at $2.5^{\prime \prime}$ distance from the central star.

\section{Results and Discussion}

Fig. 2 gives an overview of the stellar spectra in the optical (left panel) and near-IR (right panel), respectively. All identified emission lines are presented in table 1. The first column gives the wavelength position of the peak maximum in the optical (DIS) spectrum. The second column gives the species name. The third column gives the central band position of the peak maximum of the near-IR emission line (TSpec), the identification is given in column four. For the near-IR spectra we obtained an absolute flux calibration and the flux of the peak maximum is given for a first indication of relative peak strengths 
Table 1. Overview of the stellar emission lines in the DIS and TSpec spectrum of MWC 922.

\begin{tabular}{|c|c|c|c|c|}
\hline$\left|\begin{array}{c}\lambda \text { optical } \\
\mathrm{nm}\end{array}\right|$ & species name & $\| \begin{array}{c}\lambda \mathbf{I R} \\
\mu \mathrm{m}\end{array}$ & species name & $\mid \begin{array}{c}\text { Flux at Peak Maximum } \\
{\left[10^{-9} \mathrm{erg} \mathrm{s}^{-1} \mathrm{~cm}^{-2} \mu \mathrm{m}^{-1}\right]}\end{array}$ \\
\hline 4243.43 & {$[\mathrm{Fe} \quad \mathrm{II}]$} & || 1.000 & Fe II & 1.5 \\
\hline 4286.98 & {$[\mathrm{Fe}$ II $]$} & 1.005 & H I Pa (7-3) & 1.4 \\
\hline 4357.74 & {$[\mathrm{Fe}$ II $]$} & 1.017 & Fe II & 0.757 \\
\hline 4413.99 & {$\left[\begin{array}{lll}\mathrm{Fe} & \mathrm{II}\end{array}\right]$} & 1.051 & Fe II & 1.403 \\
\hline 4452.09 & {$[\mathrm{Fe}$ II $]$} & 1.086 & Fe II & 1.344 \\
\hline 4490.19 & Fe I & 1.094 & H I Pa (6-3) & 2.482 \\
\hline 4582.73 & Fe II & 1.112 & Fe II & 1.095 \\
\hline 4628.09 & {$[\mathrm{Fe}$ II $]$} & 1.129 & O I & 1.923 \\
\hline 4815.00 & {$[\mathrm{Fe}$ II $]$} & 1.252 & {$[\mathrm{Fe}$ II $]$} & 0.622 \\
\hline 4862.15 & Н I Н $\beta$ Ва $(4-2)$ & 1.257 & {$[\mathrm{Fe} \quad \mathrm{II}]$} & 2.477 \\
\hline 4905.69 & {$[\mathrm{Fe} \quad \mathrm{II}]$} & 1.270 & {$[\mathrm{Fe}$ II $]$} & 0.755 \\
\hline 4923.84 & Fe II & 1.282 & H I Pa (5-3) & 5.801 \\
\hline 5018.19 & Fe II & 1.294 & {$[\mathrm{Fe}$ II $]$} & 1.037 \\
\hline 5157.90 & {$[\mathrm{Fe}$ II $]$} & 1.316 & $\mathrm{O} \mathrm{I}$ & 1.094 \\
\hline 5168.78 & Fe II & 1.513 & H I Br (21-4) & 0.918 \\
\hline 5197.81 & Fe II & 1.519 & H I Br $(20-4)$ & 0.925 \\
\hline 5234.10 & {$[\mathrm{Fe}$ II $]$} & 1.526 & H I Br $(19-4)$ & 0.929 \\
\hline 5261.31 & {$[\mathrm{Fe}$ II $]$} & 1.533 & $\mathrm{H} \mathrm{I} \mathrm{Br}(18-4)$ and $[\mathrm{Fe}$ II] & 1.419 \\
\hline 5295.79 & $\mathrm{O} \mathrm{I}$ & 1.544 & $\mathrm{H} \mathrm{I} \mathrm{Br}(17-4)$ & 0.989 \\
\hline 5315.75 & Fe II & 1.556 & H I Br $(16-4)$ & 1.024 \\
\hline 5333.90 & {$[\mathrm{Fe}$ II $]$} & 1.570 & H I Br $(15-4)$ & 1.026 \\
\hline 5362.93 & Fe II & 1.588 & H I Br (14-4) & 1.091 \\
\hline 5411.92 & {$[\mathrm{Fe} \quad \mathrm{II}]$} & 1.599 & {$[\mathrm{Fe} \quad \mathrm{II}]$} & 1.44 \\
\hline 5431.87 & $\mathrm{Fe} \mathrm{I}$ & 1.611 & H I Br $(13-4)$ & 1.093 \\
\hline 5495.38 & Fe I & 1.641 & H I Br $(12-4)$ & 1.190 \\
\hline 5526.22 & {$[\mathrm{Fe}$ II $]$} & 1.643 & {$[\mathrm{Fe} \mathrm{II}]$} & 2.988 \\
\hline 5575.21 & {$\left[\begin{array}{ll}\mathrm{O} & \mathrm{I}\end{array}\right]$} & 1.664 & Fe II & 1.185 \\
\hline 6298.65 & O I & 1.677 & {$[\mathrm{Fe} \quad \mathrm{II}]$} & 1.451 \\
\hline 6362.83 & O I & 1.679 & H I Br (11-4) & 1.386 \\
\hline 6562.22 & Н I $\mathrm{H} \alpha$ Ba (3-2) & 1.736 & H I Br $(10-4)$ & 1.557 \\
\hline 6665.35 & {$[\mathrm{Ni}$ II $]$} & 2.165 & H I Br $(7-4)$ & 3.092 \\
\hline 7153.52 & {$\left[\begin{array}{lll}\mathrm{Fe} & \mathrm{II}\end{array}\right]$} & 2.224 & $\mathrm{H}_{2}$ & 2.023 \\
\hline 7375.83 & {$[\mathrm{Ni} \quad \mathrm{II}]$} & & & \\
\hline 7410.21 & {$[\mathrm{Ni}$ II $]$} & & & \\
\hline 7451.46 & {$[\mathrm{Fe}$ II $]$} & & & \\
\hline 7513.35 & Fe II & & & \\
\hline 7710.45 & Fe II & & & \\
\hline 8306.33 & Fe II & & & \\
\hline 8446.14 & $\mathrm{O} \mathrm{I}$ & & & \\
\hline 8466.76 & H I Pa (17-3) & & & \\
\hline 8496.56 & Ca II & & & \\
\hline 8540.10 & $\mathrm{Ca}$ II / H I Pa (15-3) & & & \\
\hline 8597.40 & H I Pa (14-3) & & & \\
\hline 8615.73 & {$[\mathrm{Fe}$ II $]$} & & & \\
\hline 8661.57 & $\mathrm{Ca}$ II / H I Pa (13-3) & & & \\
\hline 8748.66 & H I Pa (12-3) & & & \\
\hline 8863.26 & H I Pa (11-3) & & & \\
\hline 8890.76 & {$[\mathrm{Fe} \quad \mathrm{II}]$} & & & \\
\hline
\end{tabular}

in the last column. Our data shows a plethora of emission lines, including neutrals and cations and also one molecular species: $\mathrm{H}_{2}$. We clearly identify Fe II lines in addition to [Fe II]. Paschen lines, Balmer and Brackett lines are also observed in emission. A first rough comparison to HD 44179, the central object in the Red Rectangle Nebula, shows overlap in Fe II, [Fe II] and some Ca II lines (Hobbs et al. 2004). A thorough analysis of the emission bands of the Red Square Nebula obtained with X-Shooter is underway.

\section{References}

Allen, D. A. \& Swings, J. P. 1976, ApJ, 47, 293

Hobbs, L. M., Thorburn, J. A., Oka, T., et al. 2004, ApJ, 615, 947

Rudy, R. J., Erwin, P., Rossano, G. S., \& Puetter, R. C. 1992, ApJ, 398, 278

Tuthill, P. G. \& Lloyd, J. P. 2007, Science, 316, 247 